\title{
Estudo de correlação entre provas funcionais respiratórias e o teste de caminhada de seis minutos em pacientes portadores de doença pulmonar obstrutiva crônica*
}

\author{
Sérgio Leite Rodrigues ${ }^{1}$, CARlos Alberto de Assis VIEGAS²
}

Introdução: A espirometria e a gasometria são largamente utilizadas na estimativa da limitação ventilatória e do prognóstico de pacientes portadores de doença pulmonar obstrutiva crônica. Entretanto, testes físicos funcionais, como o teste de caminhada de seis minutos (Tc6), têm surgido como complemento na avaliação dinâmica de portadores de doença pulmonar obstrutiva crônica.

Objetivo: Determinar as correlações entre as provas funcionais respiratórias e o teste de caminhada de seis minutos em portadores de DPOC. Pacientes e métodos: 45 pacientes foram submetidos à

coleta de história clínica e a exame físico completo realizado pela equipe médica do Serviço de Pneumologia do Hospital Universitário de Brasília. Em seguida, foi realizada a avaliação das variáveis espirométricas, gasométricas, das pressões respiratórias e o teste de caminhada de seis minutos (Tc6).

Resultados: Observaram-se correlações positivas estatisticamente significativas $(p<0,05)$ das variáveis $\mathrm{VEF}_{1}, \mathrm{PaO}_{2}, \mathrm{SpO}_{2}$ e $\mathrm{Pe}_{\text {máx }}$ em relação ao teste de caminhada de seis minutos. Conclusões: $\mathrm{O}$ teste de caminhada de seis minutos correlacionou-se de forma significativa $(\mathrm{p}<0,05)$ e positiva $e$ pode ser utilizado como instrumento alternativo na avaliação funcional do paciente portador de DPOC.

(J Pneumol 2002;28(6):324-8)

Study of correlation between functional respiratory tests and the six minute walk test in patients with chronic obstructive pulmonary disease

Introduction: Spirometry and analysis of blood gases data have been extensively used to assess ventilatory limitation and prognosis of COPD patients. However, functional physical tests, such as the six-minute walk test (6wt) have been used for the dynamic assessment of COPD patients.

Objective: To determine correlations between respiratory function test data and the six-minute walk test in COPD patients. Patients and methods: 45 patients were submitted to clinical history survey and complete physical examination performed by the medical staff of the Pneumology Department of the Brasilia University Hospital, followed by spirometry, analysis of arterial blood gases, maximal respiratory pressures data, and the six-minute walk test. Results: Concerning the spirometric, analysis of blood gases and maximal respiratory pressures data, statistically significant positive correlations $(p<0.05)$ between $\mathrm{FEV}_{1}, \mathrm{PaO}_{2}, \mathrm{SpO}_{2}, \mathrm{MEP}$ and the sixminute walk test were obtained. Conclusions: For the studied group, the six minute walk test has significant correlation with $\mathrm{FEV}_{1}, \mathrm{PaO}_{2}, \mathrm{SpO}_{2}$ and $\mathrm{MEP}$, and can be used as an alternative functional assessment of COPD patients.

* Trabalho realizado no Laboratório de Provas de Função Pulmonar do Serviço de Pneumologia do Hospital Universitário de Brasília, da Faculdade de Medicina da Universidade de Brasília - UnB.

1. Mestre em Ciências da Saúde. Especialista em Fisioterapia Respiratória.

2. Doutor em Medicina - Pneumologia.
Endereço para correspondência - Av. Flamboyant, Quadra 105, Lote 02, apto. 801, Águas Claras - 72030-100 - Taguatinga, DF. Tel.: (61) 435-4502 (res.)/933-4616 (cel.); e-mail: sleite99@ig.com.br

Recebido para publicação em $27 / 6 / 02$. Aprovado, após revisão, em 9/10/02. 
Descritores - Pneumopatias obstrutivas. Teste de esforço. Capacidade pulmonar total. Volume residual. Volume expiratório forçado. Key words - Pulmonary rehabilitation. Obstructive lung diseases. Exercise test. Total lung capacity. Residual volume. Forced expiratory volume.

\section{INTRODUÇÃO}

O paciente portador de doença pulmonar obstrutiva crônica (DPOC) pode ter redução importante da sua capacidade física devido a vários fatores, tais como: hiperinsuflação dinâmica e aumento do metabolismo muscular glicolítico, acompanhado de descondicionamento físico progressivo associado à inatividade $e^{(1)}$. Na estimativa da limitação ventilatória e do prognóstico desses pacientes, são largamente utilizadas a espirometria e a gasometria arterial $^{(2)}$. Entretanto, testes físicos funcionais, como o teste de caminhada de seis minutos (Tc6), têm surgido como complemento na avaliação dinâmica de portadores de DPOC $^{(3)}$.

Os testes de caminhada são administrados em programas de reabilitação pulmonar (PRP) com intuito de avaliar a capacidade física, monitorar a efetividade do tratamento e estabelecer o prognóstico de pacientes com DPOC ${ }^{(4,5)}$. Nesses pacientes, devido ao comprometimento das vias aéreas e da arquitetura pulmonar, as provas funcionais apresentam deterioração importante e contínua em função do tempo. Apesar de as provas funcionais serem realizadas principalmente com o paciente em repouso, devemos salientar que a avaliação estática pode subestimar a capacidade de exercício, assim como a capacidade em realizar as atividades diárias. Esta, sim, pode refletir sua qualidade de vida, correlacionando-se com o grau de satisfação ou insatisfação do paciente com sua própria condição física ${ }^{(6,7)}$.

Embora saibamos da existência de testes funcionais dinâmicos sofisticados, como a ergoespirometria, na aferição da capacidade física de pacientes com DPOC, escolhemos o Tc6 por ser o mais utilizado no mundo para avaliação do esforço submáximo. O Tc6 é simples e de baixo custo operacional e, principalmente, reflete as atividades diárias desenvolvidas pelos pacientes portadores de DPOC ${ }^{(4,8)}$.

O principal objetivo deste estudo foi determinar as correlações entre provas de função pulmonar (gasometria, espirometria e manovacuometria) com o Tc6, bem como sua utilização na avaliação da capacidade funcional de pacientes portadores de DPOC.

\section{PACIENTES}

Realizamos um estudo transversal com pacientes portadores de DPOC, avaliados no Laboratório de Prova de
Siglas e abreviaturas utilizadas neste trabalho

CPT - Capacidade pulmonar total

CVF - Capacidade vital forçada

$\mathrm{pH}$ - O logaritmo negativo, para a base 10 , da concentração de íons livres de hidrogênio em uma solução

$\mathrm{PaCO}_{2}$ - Pressão parcial de dióxido de carbono no sangue arterial

$\mathrm{PaO}_{2}$ - Pressão parcial de oxigênio no sangue arterial

$\mathrm{Pe}_{\text {máx }}$ - Pressão expiratória máxima

$\mathrm{Pi}_{\text {máx }}$ - Pressão inspiratória máxima

$\mathrm{RV}$ - Volume residual

$\mathrm{SpO}_{2}$ - Percentagem da saturação arterial periférica da hemoglobina pelo oxigênio

Tc6 - Teste de caminhada de seis minutos

$V_{E F}$ - Volume expirado forçado no primeiro segundo

$\mathrm{VEF}_{1} / \mathrm{CVF} \%$ - Razão entre volume expiratório forçado no primeiro segundo e a capacidade vital forçada

DPOC - Doença pulmonar obstrutiva crônica

Função Pulmonar do Hospital Universitário de Brasília (HUB) no período de janeiro de 2001 a fevereiro de 2002, que haviam sido encaminhados ao Programa de Reabilitação Pulmonar pelo Serviço Ambulatorial de Pneumologia do HUB.

\section{CRITÉRIOS DE INCLUSÃO}

Foram incluídos pacientes portadores de DPOC encaminhados ao Programa de Reabilitação Pulmonar do HUB com queixas de restrições físicas e sociais devido a enfermidade, estáveis clinicamente e sem períodos de agudização da doença nas oito semanas anteriores. Todos eram medicados com bamifilina e nebulização com $\beta_{2}$-adrenérgico e anticolinérgico. O diagnóstico clínico e funcional de DPOC foi baseado na história de exposição ao fator de risco, produção de secreção, dispnéia e uma prova espirométrica alterada após o uso de broncodilatador ${ }^{(9)}$.

O protocolo de pesquisa deste trabalho foi aprovado pela Comissão de Ética do Hospital Universitário de Brasília-UnB. Todos os pacientes assinaram termo de consentimento pós-informado e foram devidamente esclarecidos.

\section{CRITÉRIOS DE EXCLUSÃo}

Foram excluídos os portadores de doença cardiovascular ou osteoarticular, que limitassem a realização segura dos testes propostos pelo protocolo.

\section{MÉTODO}

Todos os pacientes incluídos foram submetidos à coleta da história clínica e a exame físico completos, realizados pela equipe médica do Serviço de Pneumologia do HUB. Em seguida, foi realizada a avaliação das variáveis espirométricas, gasométricas, pressão inspiratória e ex- 
piratória máxima e o teste de caminhada de seis minutos (Tc6) de acordo com as descrições abaixo:

- Espirometria: foram realizadas três manobras de expiração forçada com os indivíduos na posição sentada, sendo escolhida pelo próprio aparelho a curva de melhor desempenho. As variáveis estudadas foram a CVF, $\mathrm{VEF}_{1}$ e a relação $\mathrm{VEF}_{1} / \mathrm{CVF} \%$. Foram medidos os valores absolutos e calculados os percentuais relativos aos preditos para sexo, idade e altura, seguindo tabela descrita por Knudson et al. ${ }^{(10)}$, já gravados na memória do aparelho (Vmax - 22 series spirometer SensorMedics, Yorba Linda, California, USA).

- Gasometria arterial: a amostra de sangue colhida através da punção da artéria radial do membro não dominante, após anestesia com xilocaína, com o paciente em repouso. Foram colhidos $2 \mathrm{ml}$ de sangue e imediatamente processados em um analisador de gás computadorizado e automatizado para medir os valores da $\mathrm{PaO}_{2}, \mathrm{PaCO}_{2}$ e a $\mathrm{SaO}_{2}$ expressos em mmHg e percentual de saturação, respectivamente. $\mathrm{O}$ aparelho utilizado para analisar as amostras foi o Ciba Corning 278 Gas System (Ciba Corning, Diagnostics Corp; Medifield, USA), calibrado automaticamente todas as manhãs, antes do início dos exames, segundo as normas estabelecidas.

- Pressão inspiratória e expiratória máxima $\left(\mathrm{Pi}_{\text {máx }}\right.$ e Pemáx): a pressão inspiratória máxima foi aferida após o paciente expirar da capacidade pulmonar total (CPT) até o volume residual (RV) com subseqüente esforço inspiratório máximo contra uma válvula ocluída. $\mathrm{A} P e_{\text {máx }}$ foi obtida após o paciente inspirar do $\mathrm{RV}$ até a $\mathrm{CPT}$ e subseqüente esforço expiratório máximo contra uma válvula ocluída. $\mathrm{O}$ instrumento utilizado para aferição dessas pressões foi o manovacuômetro (Criticalmed, Industries Inc, Rio de Janeiro, Brasil). Foram realizadas três manobras de $\mathrm{Pi}_{\text {máx }}$ $e e_{\text {máx }}$ com objetivo de obter o maior valor das três aceitáveis (diferença entre os valores $<5 \%$ ). Os valores do estudo foram comparados com os valores previstos de Black e Hyatt ${ }^{(11)}$.

- Tc6: consistiu na avaliação da distância máxima percorrida pelo paciente durante seis minutos. Esse procedimento foi realizado em uma pista coberta com 25 metros de comprimento. Utilizamos, durante o teste, duas frases padronizadas de incentivo ao paciente no terceiro e quinto minuto, visando o aumento do seu rendimento físico. Inicialmente cada paciente realizou dois testes em dias alternados. O primeiro teve o propósito de adaptar o paciente ao procedimento $e$ o segundo a aferir os valores do estudo. Os parâmetros aferidos antes e após o teste foram a freqüência respiratória, a freqüência cardíaca, a saturação da hemoglobina pelo oxigênio $\left(\mathrm{SpO}_{2}\right)$ e a distância total percorrida, a ser comparada com os valores propostos por Enright e Sherrill ${ }^{(12)}$.

\section{AnÁlise estaTísticA}

Os dados obtidos são apresentados em seus valores médios e desvios padrões e a análise de correlação entre as variáveis numéricas foi feita pela análise de Pearson. Consideram-se estatisticamente significativas as diferenças com $\mathrm{p}<0,05$.

\section{Resultados}

Foram encaminhados 50 pacientes para o Laboratório de Provas de Função Pulmonar. Quatro foram excluídos por cardiopatia grave e um por artropatia grave. Quarenta e cinco pacientes estudados, dos quais 40 eram do sexo masculino. A média de idade do grupo era de $66 \pm$ 8 anos, variando entre 48 e 80 anos. O índice de massa corporal (IMC) foi de $25 \pm 6 \mathrm{~kg} / \mathrm{m}^{2}$, variando entre $17 \mathrm{e}$ $47 \mathrm{~kg} / \mathrm{m}^{2}$ (Tabela 1 ).

Em relação à espirometria, observamos $\mathrm{VEF}_{1}$ médio de $46 \pm 22 \%$ do previsto, variando entre $17 \%$ e máximo de $121 \%$. A CVF foi de $70 \pm 29 \%$ do previsto, com valor mínimo de $26 \%$ e máximo de $178 \%$. Já no índice $\mathrm{VEF}_{1} /$ CVF\%, observamos o valor médio de $54 \pm 13 \%$, tendo o mínimo de $32 \%$ e o máximo de $78 \%$.

No exame gasométrico, observamos pH médio de 7,42 $\pm 0,04$, com valor mínimo de 7,3 e máximo de 7,47.

Em relação à $\mathrm{PaCO}_{2}$, obtivemos valor médio de $37 \pm$ $6 \mathrm{mmHg}$, com o valor mínimo de $25,4 \mathrm{mmHg}$ e máximo de $56,2 \mathrm{mmHg}$. A $\mathrm{PaO}_{2}$ média observada foi de $71 \pm$ $12 \mathrm{mmHg}$, com valor mínimo de $39,1 \mathrm{mmHg}$ e máximo

TABELA 1

Características demográficas e funcionais da população estudada

\begin{tabular}{|c|c|}
\hline Variáveis & Características \\
\hline Sexo & $\begin{array}{l}40 \text { - homens } \\
5 \text { - mulheres }\end{array}$ \\
\hline Idade & $66 \pm 8$ anos \\
\hline IMC & $25 \pm 6 \mathrm{~kg} / \mathrm{m}^{2}$ \\
\hline Espirometria & $\begin{array}{c}\text { CVF* }^{*}=70 \pm 29 \% \\
\operatorname{VEF}_{1}=46 \pm 22 \% \\
\mathrm{VEF}_{1} / \mathrm{CVF} \%=54 \pm 13 \%\end{array}$ \\
\hline Gasometria & $\begin{array}{c}\mathrm{pH}=7,42 \pm 0,04 \\
\mathrm{PaCO}_{2}=37 \pm 6 \mathrm{mmHg} \\
\mathrm{PaO}_{2}=71 \pm 12 \mathrm{mmHg} \\
\mathrm{SpO}_{2}=94 \pm 3 \%\end{array}$ \\
\hline Pressões respiratórias & $\begin{array}{l}\mathrm{Pi}_{\text {máx }}=-96 \pm 19 \mathrm{cmH}_{2} \mathrm{O} \\
\mathrm{Pe}_{\text {máx }}=120 \pm 10 \mathrm{~cm}_{2} \mathrm{O}\end{array}$ \\
\hline Tc6 & Distância $=530 \pm 83$ metros \\
\hline
\end{tabular}


de $95 \mathrm{mmHg}$. Em relação à saturação da hemoglobina pelo oxigênio $\left(\mathrm{SpO}_{2}\right)$, observamos valor médio de $94 \pm$ $3 \%$ com o mínimo de $81 \%$ e o máximo de $97 \%$.

$\mathrm{Na}$ aferição das pressões respiratórias, obtivemos valores médios de $-96 \pm 19 \mathrm{cmH}_{2} \mathrm{O}$ e $120 \pm 10 \mathrm{cmH}_{2} \mathrm{O}$, para a $\mathrm{Pi}_{\text {máx }}$ e $\mathrm{Pe} e_{\text {máx }}$ respectivamente. Em relação à distância total percorrida (dt) no Tc6, observamos o valor médio de 530 \pm 83 metros, com mínimo de 280 metros e máximo de 685 metros. Os valores médios evidenciados pela espirometria, gasometria arterial, aferição das pressões inspiratórias e expiratórias máximas e o Tc6 podem ser observados na Tabela 1.

Em relação à análise de correlação, observamos correlação estatisticamente significativa entre o Tc6 $e$ as seguintes variáveis: $\mathrm{VEF}_{1} \mathrm{x} \mathrm{dt}, \mathrm{PaO}_{2} \mathrm{x} \mathrm{dt}, \mathrm{SpO}_{2} \mathrm{x} \mathrm{dt}$ e $\mathrm{Pe}_{\text {máx }} \mathrm{x} \mathrm{dt}$ (Figura 1), não sendo observados outros valores significativos.

\section{DISCUSSÃO}

Neste estudo observamos correlações significativas entre variáveis funcionais respiratórias e o Tc6, quando utilizado na avaliação da capacidade física de pacientes por- tadores de DPOC. Guell et al. ${ }^{(13)}$, em estudo randomizado, observaram que o Tc6 é um instrumento válido, devendo ser considerado para avaliação funcional e manejo clínico a longo prazo do portador de DPOC. Os autores concluíram que o Tc6 foi o principal indicador de aumento da capacidade funcional entre os grupos experimental e controle, na avaliação dos efeitos da reabilitação pulmonar, em 60 pacientes avaliados sistematicamente durante dois anos.

Os resultados dos Tc6 apresentados em nosso trabalho vão ao encontro dos valores de referência para adultos saudáveis propostos por Enright e Sherrill(12). Entretanto, temos que considerar que esses valores se referem a indivíduos saudáveis realizando o Tc6 sem o encorajamento verbal, por parte do avaliador. Isso sugere que o estímulo verbal por nós adotado pode ter sido responsável pelo melhor rendimento físico apresentado pelos pacientes do estudo. Segundo Guyatt et al. ${ }^{(14)}$, durante o Tc6 a estimulação verbal determina melhor rendimento físico em pacientes portadores de enfermidades cardiopulmonares.

Por outro lado, os valores das pressões inspiratórias $e$ expiratórias máximas dos pacientes estudados também

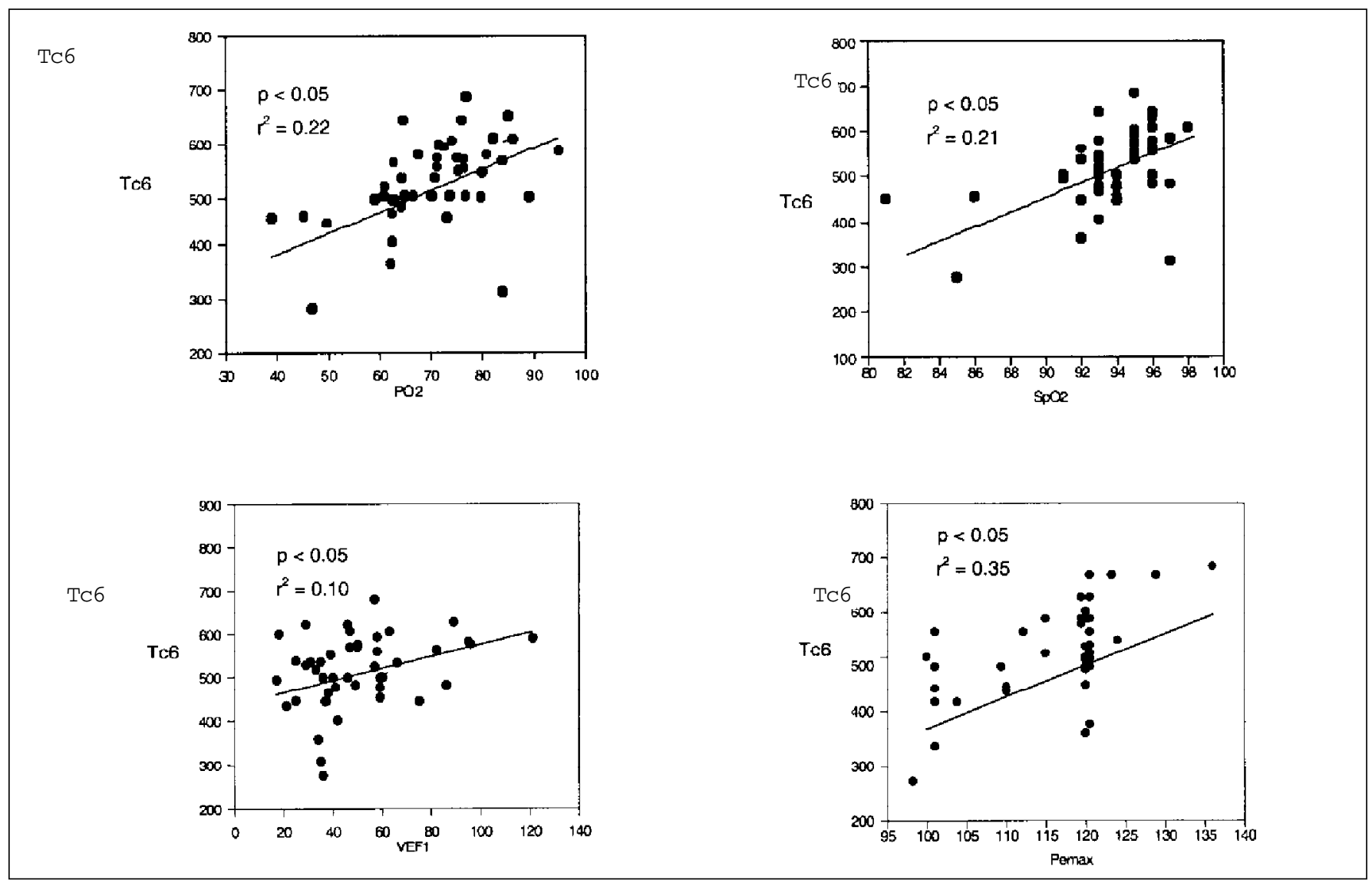

Figura 1 - Características gráficas da análise de correlação entre o teste de caminhada de seis minutos e as variáveis funcionais respiratórias da população estudada 
se apresentaram dentro dos parâmetros considerados normais ${ }^{(9)}$. Esses resultados concordam com a literatura pertinente, na qual se observam adaptações metabólicas e morfológicas das fibras musculares com a preservação da força diafragmática em pacientes com DPOC, mesmo diante do encurtamento das fibras musculares devido à hiperinsuflação ${ }^{(15)}$.

A correlação entre o Tc6 e parâmetros como sobrevida, função pulmonar, qualidade de vida e capacidade cognitiva já vem sendo discutida amplamente pela literatu$\mathrm{ra}^{(1,6,16)}$. Em estudo prospectivo, Bowen et al. ${ }^{(17)}$ afirmaram que os pacientes portadores de DPOC que apresentam maior distância percorrida no Tc6 possuem maior sobrevida, em relação aos que têm pior rendimento neste teste. Ainda mais, o Tc6 foi considerado melhor preditor de sobrevida que os parâmetros tradicionais, como a necessidade da oxigenioterapia a longo prazo e a gravidade da obstrução ao fluxo aéreo avaliado pelo $\mathrm{VEF}_{1}$. Em nosso estudo, o Tc6 correlacionou-se de forma significativa ( $\mathrm{p}<$ $0,05)$ com o $\mathrm{VEF}_{1}$, sugerindo que a utilização do Tc6 pode auxiliar na avaliação da deterioração da função pulmonar, da sobrevida e do nível de funcionalidade física dos portadores de DPOC. Para Casaburi(18), a redução das fibras musculares do tipo I, da densidade capilar e, principalmente, o aumento no desequilíbrio entre a demanda e oferta de oxigênio muscular determinam a redução do metabolismo aeróbio local em pacientes portadores de DPOC. Isso vem corroborar outra correlação encontrada no presente estudo, entre a distância percorrida no Tc6 $e$ a $\mathrm{PaO}_{2}(\mathrm{p}<0,05)$ e $\mathrm{SpO}_{2}(\mathrm{p}<0,05)$. Esse dado sugere que a ação do metabolismo aeróbio, mantida pelo oxigênio disponível para a musculatura esquelética, pode ter reduzido os efeitos deletérios do desequilíbrio entre a demanda $e$ a oferta de oxigennio durante o Tc6 dos pacientes estudados. A interessante correlação encontrada entre a $\mathrm{Pe}_{\text {máx }}(\mathrm{p}<0,05)$ e o Tc6 pode ser traduzida como uma adaptação da musculatura expiratória frente às alterações mecânicas dos músculos ventilatórios, decorrentes da hiperinsuflação pulmonar. Segundo Gáldiz Iturri ${ }^{(15)}$, em pacientes com obstrução grave ao fluxo aéreo, a contração da musculatura expiratória abdominal determina elevação e redução do diâmetro das cúpulas diafragmáticas, otimizando a relação comprimento-tensão do diafragma. Esse auxílio favorece a contração diafragmática, a ventilação pulmonar e, conseqüentemente, a capacidade física do paciente portador de DPOC.

Este estudo apresenta limitações que devem ser consideradas. A espirometria não contemplou os volumes pulmonares e a capacidade de difusão do monóxido de carbono $\left(\mathrm{D}_{\mathrm{L}} \mathrm{CO}\right)$, sabidamente, importantes fatores relacionados ao desempenho físico na $\mathrm{DPOC}^{(1,15)}$.

Finalmente, podemos concluir que o presente estudo demonstrou que o Tc6 é um instrumento que se correla- ciona significantemente com indicadores de prognóstico da DPOC, ou seja, $\mathrm{VEF}_{1}$ e $\mathrm{PaO}_{2}$. O Tc6 pode ser utilizado como um método alternativo na aferição da capacidade funcional de pacientes portadores de DPOC avaliados $e$ tratados em nosso meio.

\section{REFERÊNCIAS}

1. Marin JM, Carrizo SJ, Gascon M, Sanchez A, Gallego B, Celli BR. Inspiratory capacity, dynamic hyperinflation, breathlessness and exercise performance during the 6-minutes-walk test in chronic obstructive pulmonary disease. Am J Respir Crit Care Med 2001;163:1395-9.

2. Rejeski WJ, Foley KO, Woodard CM, Zaccaro DJ, Berry MJ. Evaluating and understanding performance testing in COPD patients. J Cardiopulm Rehabil 2000;20:79-88.

3. Van Stel HF, Bogaard JM, Rijssenbeek-Nouwens LH, Colland VT. Multivariable assessment of the 6-min walking test in patients with chronic obstructive pulmonary disease. Am J Respir Crit Care Med 2001;163: 1567-71

4. Solway S, Brooks D, Lacasse Y, Thomas S. A qualitative systematic overview of the measurement properties of walk tests used in the cardiorespiratory domain. Chest 2001;119:256-70.

5. Rodrigues SL, Viegas CAA, Lima T. Efetividade da reabilitação pulmonar como tratamento coadjuvante da doença pulmonar obstrutiva crônica. J Pneumol 2002;28:65-70.

6. De Torres JP, Pinto-Plata V, Ingenito E, Bagley P, Gray A, Berger R, et al. Power of outcome measurement to detect clinically significant changes in pulmonary rehabilitation of COPD. Chest 2002;121:1092-8.

7. Garrod R, Bestall JC, Paul EA, Wedzicha JA, Jones PW. Development and validation of a standardized measure of activity of daily living in patients with severe COPD: the London Chest Activity of Daily Living scale (LCADL). Respir Med 2000;94:589-696.

8. Moreira MAC, Moraes MR, Tannus R. Teste de caminhada de seis minutos em pacientes com DPOC durante programa de reabilitação. J Pneumol 2002;27:295-300.

9. Pauwels RA, Buist AS, Calverley PM, Jenkins CR, Hurd SS. Global strategy for the diagnosis, management and prevention of chronic obstructive pulmonary disease. NHLBI/WHO Global Initiative for Chronic Obstructive Lung Disease (GOLD) Workshop summary. Am J Respir Crit Care 2001;163:1256-76.

10. Knudson RJ, Lebowitz MD, Holbverg CJ, Burrows B. Changes in the normal expiration flow-volume curve with growth and aging. Am Rev Respir Dis 1983;127:725-34.

11. Black LF, Hyatt RE. Maximal respiratory pressures: normal values and relationship to age and sex. Am Rev Respir Dis 1969;99:969-74.

12. Enright PL, Sherrill DL. Reference equations for the six-minutes walk in healthy adults. Am J Respir Crit Care Med 1998;158:1384-7.

13. Guell R, Casan P, Belda J, Sangenis M, Morante F, Guyatt GH, Sanchis J. Long-term effects of outpatient rehabilitation of COPD. Chest 2000;117:976-83.

14. Guyatt GH, Pusgley SO, Sullivan MJ, Thompson PJ, Berman LB, Jones $\mathrm{NL}$, et al. Effect of encouragement on walking test performance. Tho$\operatorname{rax} 1984 ; 39: 818-22$

15. Gáldiz Iturri JB. Función de los músculos respiratorios en la EPOC. Arch Bronconeumol 2000;36:275-85

16. Etnier J, Johnston R, Dagenbach D, Pollard RJ, Rejeski J, Berry M. The relationships among pulmonary function, aerobic fitness and cognitive functioning in older COPD patients. Chest 1999;116:953-60.

17. Bowen JB, Votto JJ, Thrall RS, Haggerty MC, Stockdale-Woolley R, Bandyopadhyay $\mathrm{T}$, et al. Functional status and survival following pulmonary rehabilitation. Chest 2000;118:697-703.

18. Casaburi R. Skeletal muscle function in COPD. Chest 2000;117:267s271 s. 